CASE REPORT

\title{
Invasive squamous cell carcinoma of the hand in a patient with Kindler syndrome: Case report and literature review
}

\author{
Etienne Cardin-Langlois MD ${ }^{1}$, Dominique Hanna MD FRCPC ${ }^{2}$, Maxime St-Amant ${ }^{3}$, Fréderic Croteau MD FRCSC
}

E Cardin-Langlois, D Hanna, M St-Amant, F Croteau. Invasive squamous cell carcinoma of the hand in a patient with Kindler syndrome: Case report and literature review. Can J Plast Surg 2010;18(3):e41-e43.

Kindler syndrome is a rare, autosomal, recessive genodermatosis characterized by trauma-induced acral blisters in infancy and childhood, photosensitivity and progressive poikiloderma. Very few cases in the literature report an association with squamous cell carcinoma, even though it is a very well-known, long-term complication. A case involving a 23 -year-old woman with a history of Kindler syndrome who was admitted to the department of plastic surgery (Sherbrooke University, Sherbrooke, Quebec) with an extensive ulcerated squamous cell carcinoma of the right hand is presented. A local excision of the tumour was initially performed, but positive margins and clinically palpable axillary lymphadenopathy over the course of hospitalization necessitated below-elbow amputation and lymph node dissection. To the authors' knowledge, this is the second reported case of aggressive metastatic squamous cell carcinoma of the hand in a patient with Kindler syndrome.

Key Words: Aggressive squamous cell carcinoma; Bullous epidermolysis; Kindler syndrome; Photosensitivity; Poikiloderma

\section{CASE PRESENTATION}

A 23-year-old woman with a history of Kindler syndrome was referred to the department of plastic surgery (Sherbrooke University, Sherbrooke, Quebec) with an ulcerated vegetating tumour in her right hand, which was progressive over a sixmonth period. The patient was from Bosnia and the diagnosis of Kindler syndrome was made at 11 years of age. She had a history of acral blistering in infancy and childhood, progressive photosensitivity and occasional gingivitis. She was treated at 18 years of age for esophageal stenosis and at 22 years of age with labiaplasty for vulva malformation. The patient reported no recent history of asthenia or increased fatigue. On physical examination, she had fragile 'cigarette paper-like' skin with aged appearance, palmoplantar hyperkeratosis and poikiloderma. She also had bilateral syndactylism of the second, third and fourth interdigital spaces. As for the lesion, it covered the radial portion of her right hand palm and it extended to the proximal phalanges of the second and third digits, restricting range of movement of the latter. The lesion measured approximately $8 \mathrm{~cm} \times 6 \mathrm{~cm} \times 3 \mathrm{~cm}$ (Figure 1 ). There was no neurovascular injury. There were verrucous-like lesions on the dorsum of the second and third digits of the same hand.

Magnetic resonance imaging showed a polylobulated mass with the following diameters: $8.1 \mathrm{~cm}$ craniocaudal, $6.1 \mathrm{~cm}$
Carcinome épidermoïde invasif de la main chez une patiente souffrant du syndrome de Kindler : Rapport de cas et revue de la littérature

Le syndrome de Kindler est une génodermatose autosomique récessive rare, caractérisée par des lésions acrales secondaires à un traumatisme chez le nourrisson et l'enfant, par la photosensibilité et la poïkilodermie progressive. Dans la littérature, très peu de cas mentionnent un lien avec le carcinome épidermoïde, même s'il s'agit d'une complication à long terme très bien connue. Les auteurs présentent ici le cas d'une femme de 23 ans ayant des antécédents de syndrome de Kindler qui a été admise au service de chirurgie plastique (Centre hospitalier universitaire de Sherbrooke, Sherbrooke, Québec), pour un volumineux carcinome épidermoïde ulcéré à la main droite. Après exérèse locale initiale de la tumeur, la présence de marges positives et de lymphadénopathie axillaire cliniquement palpable durant l'hospitalisation ont forcé l'amputation sous le coude et le curage des ganglions lymphatiques. À la connaissance de l'auteur, il s'agit du second cas rapporté de carcinome épidermoïde métastatique agressif de la main associé à un syndrome de Kindler.

\footnotetext{
${ }^{1}$ Department of Surgery, Division of Plastic Surgery, McGill University, Montreal, and Sherbrooke University; ${ }^{2}$ Department of Medicine, Sherbrooke University and Division of Dermatology, Centre hospitalier universitaire de Sherbrooke, Sherbrooke, Quebec; ${ }^{3}$ Sherbrooke University, Centre hospitalier universitaire de Sherbrooke; ${ }^{4}$ Department of Surgery, Division of Plastic Surgery, Sherbrooke University, Centre hospitalier universitaire de Sherbrooke, Sherbrooke, Quebec

Correspondence: Dr Fréderic Croteau, Department of Surgery, Division of Plastic Surgery, Sherbrooke University, Centre hospitalier universitaire de Sherbrooke, 580 Bowen sud street, Sherbrooke, Quebec J1G 2E8. Telephone 819-822-0442, fax 819-822-0646,

e-mail fredcroteau@videotron.ca
} 


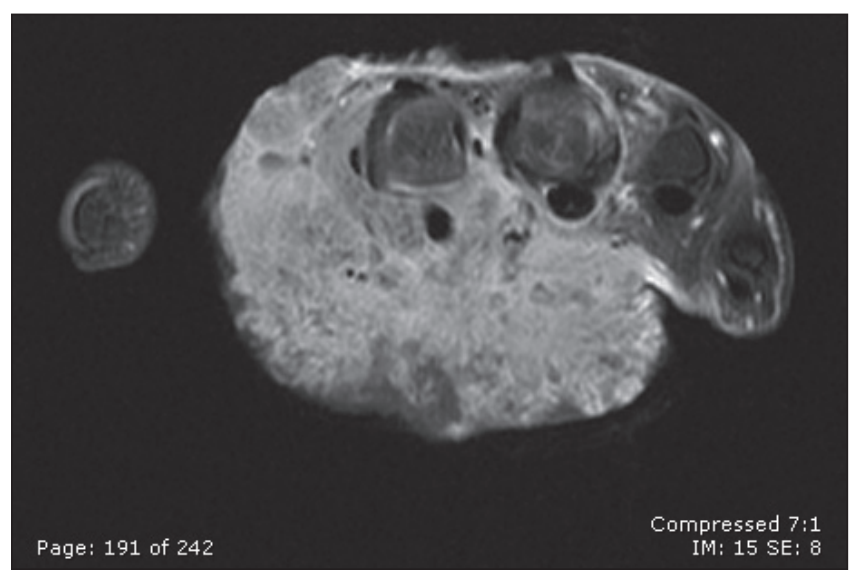

Figure 2) Magnetic resonance imaging of the transverse side of the right hand. Note the involvement of the first and second web space and the dorsal extension at the index finger. The intrinsic muscles of the index and long finger are also indistinguishable from the lesion

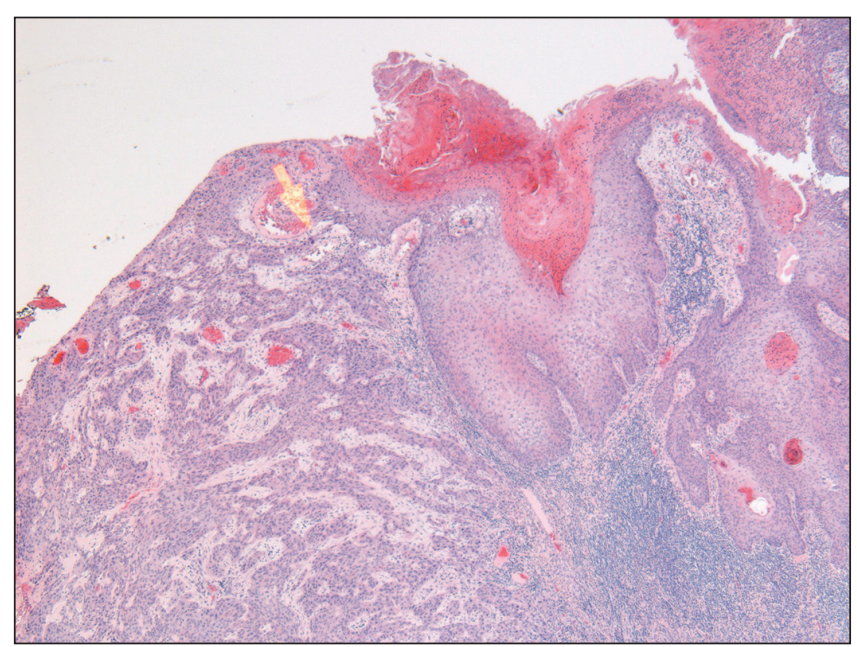

Figure 3) In situ and invasive (arrow) squamous cell carcinoma. Hematoxylin and eosin stain, original magnification $\times 4$

covered by the lesion to its dorsum. The lesion included flexor tendons and lumbricals of the second and third radius until the palmar cortex. It was placed side by side with the fourth radius until the flexor tendons. The first and fifth digits were spared from the lesion. The neurovascular bundle was indistinguishable from the lesion (Figure 2).

The authors proceeded with surgical amputation of the right hand's first and second radius. The margins were approximated without complete closure. The pathology report of the main surgical specimen showed a moderately differentiated squamous cell carcinoma with surface ulceration infiltrating all planes of muscles, fascias and bones. There were many signs of vascular emboli and the presence of peripheral nervous sheating (Figure 3). Cutaneous excision margins were negative for tumour involvement. However, profound margins were positive for the fourth digit and at less than $2 \mathrm{~mm}$ of the first digit. After discussion with the patient and a multidisciplinary team, a below-elbow surgical amputation was considered to be the best option. At the time of operation, clinical recurrence was already visible on the wound margin.

After the first surgery, a right axillary adenopathy was found on physical examination. Positron emission tomography (PET) scan indicated an axillary lymphadenopathy of $2.4 \mathrm{~cm} \times 2.1 \mathrm{~cm}$,

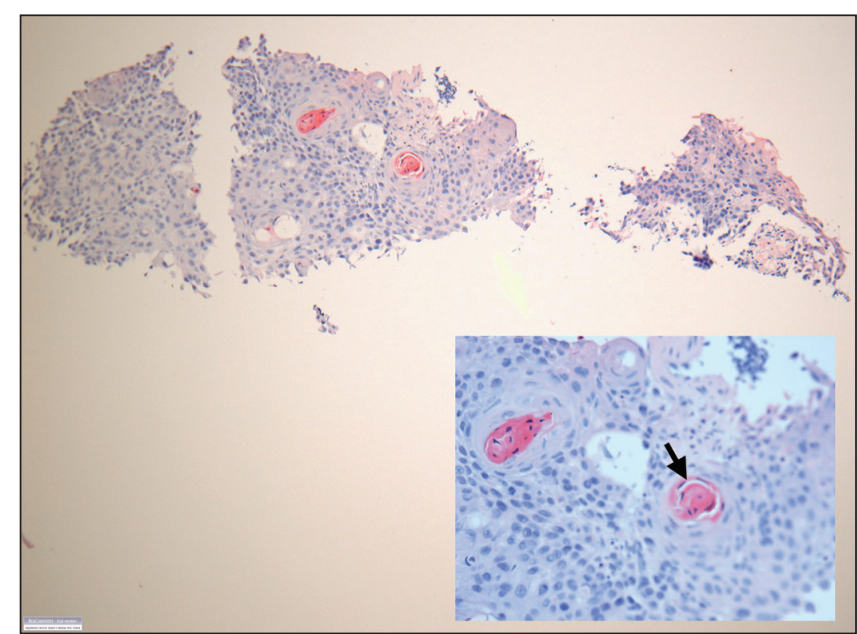

Figure 4) Keratin pearl (arrow) in metastatic squamous cell carcinoma in the right axillary lymph node. Hematoxylin and eosin stain, original magnifications $\times 10$ and $\times 20$

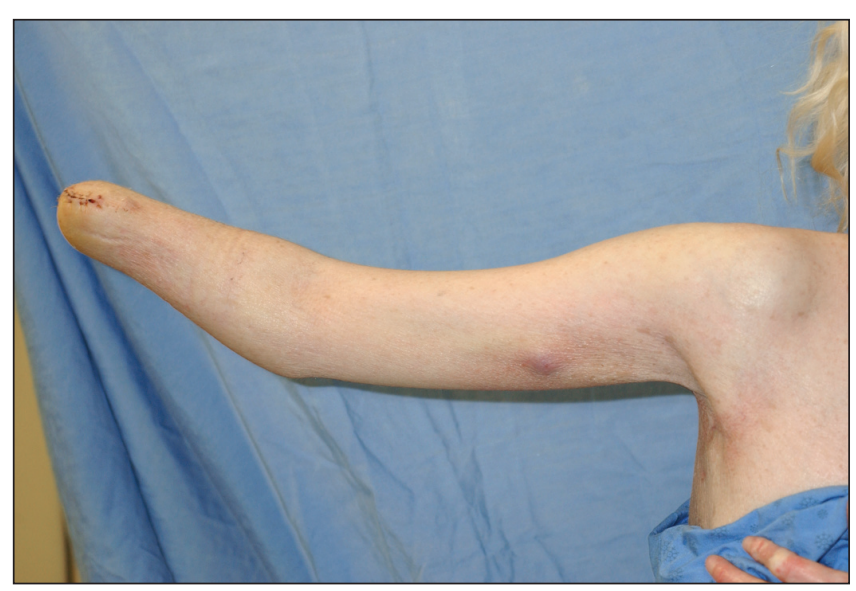

Figure 5) In-transit arm metastasis

without other associated distant metastasis. A needle aspiration biopsy was followed by an axillary lymph node dissection. The pathology report then showed one positive lymph node out of eight containing a well-differentiated keratinizing metastatic pavimentous carcinoma (Figure 4).

A surgical specimen taken on the dorsal surface of the index showed verruciform actinic keratosis with a suspicious area of squamous cell carcinoma in situ. The patient had an identical lesion removed in 2004 that was initially described as a verruca vulgaris. After reviewing the 2004 pathology slides, it was discovered that the initial lesion was more compatible with a verruciform actinic keratosis with microinvasive transformation into squamous cell carcinoma.

A few weeks after amputation and axillary lymph node dissection, multiple in-transit forearm and arm metastasis were identified on physical examination and confirmed on PET scan and biopsy PET scan (Figure 5). The present complex case was discussed with the tumour board. This extremely aggressive and rapidly progressive cutaneous squamous carcinoma in a patient with Kindler syndrome has a very poor prognosis. Because patients with this syndrome have a DNA reparation defect, radiotherapy and the majority of chemotherapy agents are contraindicated. Moreover, even aggressive chemotherapy would only be palliative. 


\section{DISCUSSION}

Kindler syndrome was first described in 1954 by Theresa Kindler (1). Approximately 100 cases have now been reported worldwide. It is an autosomal, recessive, genodermatosis characterized by photosensitivity, progressive poikiloderma, trauma-induced acral blisters in infancy and childhood, gingival fragility and cutaneous atrophy. Bullous lesions start early in childhood but tend to progressively slow down compared with poikiloderma, which gets more pronounced with age. The main differential diagnostics are congenital bullous diseases such as dystrophic bullous epidermolysis, congenital poikiloderma or RothmundThomson syndrome, and hereditary acrokeratosis poikiloderma or Weary's syndrome (2-8). There are no known associations between Kindler syndrome and ethnicity or sex.

There are many different manifestations of Kindler syndrome. Among these are telangiectasia, onychodystrophy, lip leukokeratosis, palmoplantar keratosis, pseudoainhum (annular constrictions around the digits, limbs or trunk) and chronic oral mucosa inflammation. Moreover, stenosis of urethral, anal or esophagal mucosa along with syndactylism have been reported (9). Actinic keratosis and squamous cell carcinoma have been described a few times, presenting particularly on the lips (6), soft palate (7) and hands $(6,8)$. These tumours are usually extremely aggressive. To our knowledge, this is the second case of metastatic aggressive squamous cell carcinoma of the hand reported in the medical literature (8). Other tumours have been associated with this syndrome including osteoma of the cranium, Wilms' tumour and transitional carcinoma of the bladder $(6,10)$.

Kindler syndrome skin histopathology is characterized by epidermal atrophy, dermoepidermal junction edema and vacuolization of the epidermis basal layer (11). A cleavage plane may be present between the basal keratinocytes and the lamina lucida, with extensive reduplication of the lamina densa (12). These characteristics, although not diagnostic, can differentiate Kindler syndrome from other bullous disease. Fermitin family homologue 1 (FFH1) protein is encoded by the FERMT-1 gene (previously known as the KIND-1 gene). This FFH1 protein, which is lacking in Kindler syndrome, normally links the actin cytoskeleton to the extracellular matrix. For other bullous diseases, the problem lies with the keratin cytoskeleton linking to the extracellular matrix (2). The FFH1 protein also plays a role in keratinocyte proliferation, adhesion and migration. Cutaneous atrophy is caused by apoptosis and the proliferation defect (3).
Moreover, although Kindler syndrome pathophysiology is still not fully elucidated, it is known that the FERMT-1 gene is involved in carcinogenesis of certain neoplasms (4). This could partly explain the frequency of these abnormalities in Kindler syndrome. A DNA reparation defect is also involved in the pathogenesis.

Genetic screening is the gold standard for Kindler syndrome diagnosis. Searching for the FFH1 gene can help in making the distinction between Kindler syndrome and other genodermatosis. Immunofluorescence microscopy with anti-FFH1 antibody can be a useful diagnostic tool (2).

Treatment of Kindler syndrome is symptomatic and aimed at prevention. Patients should avoid sun exposure and use external photoprotection to limit the progression of poikiloderma and the risk of cutaneous carcinoma. Trauma should also be avoided to reduce blister formation, which can progress into disabling scars (6). Patients can expect to live relatively normal lives if they have close follow-up visits. Blisters tend to fade after childhood, usually without scars. However, poikiloderma and skin atrophy progresses over a period of time. Complications of Kindler syndrome, including neoplasms, may limit life expectancy.

\section{CONCLUSION}

Kindler syndrome is a rare genodermatosis with significant implications for affected patients. Differential diagnosis includes bullous congenital disease and poikiloderma with photosensitivity. Prevention is important to lower ultraviolet radiation exposure and trauma. The presence of squamous cell carcinoma results in severe complications such as those reflected in our case. In lesions suspected for squamous cell carcinoma, close follow-up visits with complete anamnesis, physical examination and appropriate laboratory/radiological workup should be performed. The metastatic potential of the squamous cell carcinoma should prompt early diagnosis and management. Moreover, a warty lesion in patients with Kindler syndrome can possibly hide an in situ squamous cell carcinoma. Thus, any skin lesion in a patient with Kindler syndrome should evoke a high degree of suspicion and should be evaluated early.

ACKNOWLEDGEMENT: The authors thank the Pathology Department, Centre Hospitalier Universitaire de Sherbrooke, Sherbrooke, Quebec, for their participation.

\section{REFERENCES}

1. Kindler T. Congenital poikiloderma with traumatic bulla formation and progressive cutaneous atrophy. Br J Dermatol 1954;66:104-11.

2. Lai-Cheong JE, Tanaka A, Hawche G, et al. Kindler syndrome: A focal adhesion genodermatosis. Br J Dermatol 2008;160:233-42.

3. Herz C, Aumailley M, Schulte C, Schlötzer-Schrehardt U, Bruckner-Tuderman L, Has C. Kindlin-1 is a phosphoprotein involved in regulation of polarity, proliferation, and motility of epidermal keratinocytes. J Biol Chem 2006;281:36082-90.

4. Kloeker S, Major MB, Calderwood DA, Ginsberg MH, Jones DA, Beckerle MC. The Kindler syndrome protein is regulated by transforming growth factor-beta and involved in integrin-mediated adhesion. J Biol Chem 2004;279:6824-33.

5. Haber RM, Hanna WM. Kindler syndrome. Clinical and ultrastructural findings. Arch Dermatol 1996;132:1487-90.

6. Ashton GH, McLean WH, South AP, et al. Recurrent mutations in kindlin-1, a novel keratinocyte focal contact protein, in the

autosomal recessive skin fragility and photosensitivity disorder, Kindler syndrome. J Invest Dermatol 2004;122:78-83.

7. Lotem M, Raben M, Zeltser R, et al. Kindler syndrome complicated by squamous cell carcinoma of the hard palate: Successful treatment with high-dose radiation therapy and granulocyte-macrophage colony-stimulating factor. Br J Dermatol 2001;144:1284-6.

8. Emanuel PO, Rudikoff D, Phelps RG. Aggressive squamous cell carcinoma in Kindler's syndrome. Skinmed 2006;5:305-7.

9. Fischer IA, Kazandjieva J, Vassileva S, Dourmishev A. Kindler syndrome: A case report and proposal for clinical diagnostic criteria. Acta Dermatovenerol Alp Panonica Adriat 2005;14:61-7.

10. Alper JC, Baden HP, Goldsmith LA. Kindler's syndrome. Arch Dermato 1978;114:457.

11. Sentürk N, Usubütün A, Sahin S, et al. Kindler syndrome: Absence of definite ultrastructural feature. J Am Acad Dermatol 1999;40:335-7.

12. Ashton GHS. Kindler syndrome. Clin Exp Dermatol 2004;29:116-21. 